\title{
Nodal staging score and adequacy of nodal staging
}

This article was published in the following Dove Medical Press journal:

OncoTargets and Therapy

\section{Hui-Min Chen \\ Ge Feng}

Nanjing Jiangbei People's Hospital, Nanjing 220000, People's Republic of China
Correspondence: Ge Feng Nanjing Jiangbei People's Hospital, 552 Ge-guan Road, Nanjing 220000, Jiangsu, People's Republic of China Tel +86025 57793705 Email 825745226@qq.com
Aims: The number of lymph nodes (LNs) excised in patients with pathologic N0 is limited, and it is very likely that there will be recessive node disease after surgery, so they are at risk of understaging. The purpose of the present study is to develop a nodal staging score (NSS) in a mathematical way to assess the likelihood that a pathologic N0 gastric cancer (GCa) patient has, indeed, no occult nodal disease after surgery.

Patients and methods: A total of 14,033 stage I-III GCa patients were identified from Surveillance, Epidemiology and End Results database for analysis. A beta-binomial model was fitted to calculate the probability of missing a nodal disease. This probability is then used to calculate the NSS.

Results: The probability of missing a nodal disease is decreased with increasing LNs examined across all pT stages. Seven and 24 LNs removed and examined was enough for an NSS of 90\% in pT1 and pT2 patients, respectively, ensuring a high confidence of correct nodal negative classification. Twenty-three and $31 \mathrm{LNs}$ examined in $\mathrm{pT} 3$ and $\mathrm{pT} 4$ patients could also maintain the NSS at $80 \%$, respectively. NSS had a significant impact on patients' survival across all pT stages (all $P \mathrm{~s}<0.0001$ ).

Conclusion: The probability that GCa patients are free of true nodal disease could be provided by NSS-based prediction, which is conducive to postoperative decision and survival surveillance. In addition, NSS can define a subtle standard on how many LNs examined are enough for adequate staging dependent on pT stages. However, at least 16 LNs examined is the standard recommendation to date.

Keywords: gastric cancer, nodal-negative classification, adequate staging, prognosis

\section{Introduction}

Pathologic lymph node (LN) status is critical for resectable gastric cancer (GCa) patients, because LN metastasis is a strong risk factor for disease recurrence. In recent years, platinum and fluorouracil-based adjuvant chemotherapies, which have largely improved patients' survival, are recommended as a standard therapy for patients with resectable GCa. ${ }^{1-3}$ Considering the achievement for adjuvant chemotherapies in GCa, an accurate nodal staging system is extremely important to avoid inadequate care. However, according to the 7th Edition American Joint Committee on Cancer (AJCC) staging guideline, all pathologic nodal-negative patients are staged as $\mathrm{pN} 0$, even a small number of LNs are examined. Up to now, it is lack of efficient tool to assess the adequacy of nodal-negative classification for these patients staged as pN0.

There is no consensus on how many LNs should be swept for an accurate staging. Based on previous randomized trials, it is recommended that standardized extended (D2) lymphadenectomy with at least 16 LNs to be removed and examined could lead to adequate staging and better clinical outcomes. ${ }^{4-6}$ However, this cutoff-based recommendation is a one-size-fit-all approach and need to be carefully used in clinical practice. Moreover, previous studies have had implications on the understaging for 
pN0 GCa patients that lead to LN recurrence. ${ }^{7,8}$ Therefore, in regard to postoperative clinical decision, it is highly necessary to develop a tool to take the probability of false-negative findings of LN status into account to predict the true nodal status for pN0 patients.

The nodal staging score (NSS) is the probability that a $\mathrm{pN} 0$ patient is indeed free of nodal disease, in other words, the probability that a $\mathrm{pN} 0$ patient has no occult disease after surgery. ${ }^{9,10}$ Therefore, on the other hand, 1-NSS is an estimate of the risk of harboring occult positive LNs for a pN0 patient. In this study, with a relatively large sample size and multiple registry-derived patients from the Surveillance, Epidemiology and End Results (SEER) database, we first present a case in which NSS as a function of the number of LNs examined and patients' pT stage can be applied to predict the true nodal status for $\mathrm{pN} 0 \mathrm{GCa}$ patients.

\section{Materials and methods}

\section{Ethics statement}

The data from the SEER database which was designed and maintained by the National Cancer Institute. Research was limited to secondary use of information previously collected in the course of normal care and data were anonymized before conducting the statistical analyses. This article does not contain any studies with human participants or animals performed by any of the authors.

\section{Study population}

The SEER database publishes the population-based incidence and survival data of cancer patients, covering about $30 \%$ of cancer registries from the USA. Clinical information provided by the SEER database largely facilitates clinical cancer research. According to the 7th Edition AJCC staging guideline, GCa patients with primary stage I-III resectable tumors from SEER database were included. If tumors were staged by the AJCC 6th staging system as provided by the SEER database, two dependent authors would re-stage them based on the 7 th Edition AJCC staging guideline. For the current analysis, lymphoepithelial carcinoma $(8,082 / 3)$, gastrointestinal stromal tumors $(8,936 / 3)$, pseudosarcomatous cell carcinoma $(8,033 / 3)$, and neuroendocrine tumors $(8,013 / 3,8,244 / 3$, and 8,246/3) were excluded.

\section{Statistical analysis}

Step I: Data simulation of missing a LN during surgery

To precisely assess the probability of false-negative findings - all LNs examined were negative for a factually nodal-positive patient - by a beta-binomial model, we performed a data simulation of missing an LN that should have been detected using a part of nodal-positive patients randomly selected (eg, if a patient had $n$ LNs examined, we performed a simulation of one $\mathrm{LN}$ being missed, and therefore only $n-1$ LNs were examined). First, we generated a randomized dataset to be used for simulation for every patient, by including the top $20 \%$ and $40 \%$ of patients with pT $1-2$ and pT3-4, respectively. Then, assuming that $n$ was the number of total LNs examined and $i$ was the number of positive LNs for a patient, positive LNs for a patient in the simulation dataset were numbered 1 to $i$, and negative LNs were numbered $i+1$ to $n$. Finally, we generated a randomized positive integer termed $r x$, which was distributed uniformly, from 1 to $n$. Therefore, if $r x \leq i$, the corresponding patient was simulated by missing a positive LNs detected. Otherwise if $r x>i$, the corresponding patient was simulated by missing a negative LNs detected. In combination with the simulated dataset, the data for the rest of nodal-positive patients were directly fitted in a beta-binomial model without further simulation.

Step 2: Fitting a beta-binomial model to calculate the probability of false-negative findings of nodal status due to missing a nodal disease

Relevant algorithm has been reported by previous studies, ${ }^{9-12}$ in which a beta-binomial model was used as follows:

$$
\left(\begin{array}{l}
n \\
i
\end{array}\right) \frac{B(i+\alpha, n+\beta-i)}{B(\alpha, \beta)}
$$

In fitting this model, $n$ was the number of total LNs examined, and $i$ was the number of positive LNs for a patient. Moreover, $n$ and $i$ were both mutually independent among patients. Then, $k=n-i$ was the number of negative LNs for the patient. Assuming that this model was fitted by a given data including $N$ patients, for jth patient they are respectively expressed as $n_{j}, i_{j}$, and $k_{j}$ and which should satisfy the condition $1 \leq k_{j}<n_{j}, j=1,2 \ldots N$.

The two parameters, $\alpha$ and $\beta$, in the above model were estimated by using the maximum likelihood method, and then based on the above fit, the probability of all LNs examined was negative $(n=k, i=0)$ for a factually nodal-positive patient due to missing a nodal disease can be obtained as follows:

$$
P\left(F N_{k}\right)=P\left(i_{j}=0 \mid k, \alpha, \beta\right)=\frac{B(\alpha, k+\beta)}{B(\alpha, \beta)}
$$

In use of this model, we assumed that $n=k$, where, $B(\cdot)$ was the function of beta distribution. This model should be 
used under two assumptions: the first was the false-negative assumption in nodal-positive patients, the positive nodes might be missed due to a limited LNs resected during surgery. The second assumption was that there was no false-positive nodal staging, because we acquiesced that there was no pathologic technology matter in discriminating a nodal disease.

\section{Step 3: Prevalence of nodal disease adjusted} by false-negative probability

We adjusted the apparent prevalence of nodal disease, which was an underestimate, based on the false-negative probability as calculated above. Because the pT stage was an important clinical factor in the AJCC-based staging guideline, it was also taken into consideration as a stratified factor for computing the adjusted prevalence. Two steps were needed to calculate it.

$$
\# F N_{k}=\frac{P\left(F N_{k}\right) * \# T P_{k}}{\left[1-P\left(F N_{k}\right)\right]}
$$

where $T P_{k}$ and $F N_{k}$ were the number of true nodal-positive patients and false nodal-negative patients for a given $k$, which was the total number of LNs resected.

$$
\operatorname{Prev}(T)=\frac{\sum_{k}\left(F N_{k}+T P_{k}\right)}{\sum_{k}\left(F N_{k}+T P_{k}+T N_{k}\right)}
$$

$\operatorname{Prev}(T)$ was the adjusted prevalence of nodal disease stratified by the $\mathrm{pT}$ stage, and $T N_{k}$ was the number of true nodal-negative patients. Similarly, the adjusted prevalence was also calculated under the assumption that there were no false-positive findings of nodal staging.

\section{Step 4: Computing the NSS}

$$
N S S_{k}=\frac{1-\operatorname{Prev}(T)}{1-\operatorname{Prev}(T)+\left[\operatorname{Prev}(T) * P\left(F N_{k}\right)\right]}
$$

NSS, derived from the above formula, had two meanings. The first was at population-based level: the proportion of true nodal-negative patients in the population of pN0 patients; and the second was at individual-based level: a novel estimate for the level of confidence in the judgment of true nodalnegative for a $\mathrm{pN} 0$ patient. On the other hand, 1 - NSS was the extent of underestimate that a nodal-positive patient was staged as pN0. The individual-based level of meaning had a more prominent clinical significance and was the focus of the present study.
All statistical analyses were performed by using SAS (version 9.4, University of North Carolina, Cary, NC, USA) and R (version 3.2.3, R Foundation, Vienna, Austria).

\section{Results \\ Patients}

Characteristics of the patients are presented in Table 1 . A total of 14,033 stage I-III GCa patients were included in the analysis. Among these patients, 8,351 (59.51\%) patients were nodal positive $(\mathrm{pN}+)$ and the remaining were those who have no positive LNs detected and were staged as pN0. Obviously, based on the worldwide surgery experience from SEER database, LNs removed in pT3-4 patients were significantly more than in pT1-2 patients $(P<0.001)$.

\section{The probability of false-negative findings of nodal staging due to missing a nodal disease}

The $\alpha$ and $\beta$ in the fitted beta-binomial model across different pT stages are listed in Table 2. As expected, the probability of false-negative findings of LN status decreased as the increase of LNs removed across all $\mathrm{pT}$ stages, due to missing a nodal

\begin{tabular}{|c|c|}
\hline & No (\%) \\
\hline \multicolumn{2}{|c|}{ Pathologic T-stage } \\
\hline $\mathrm{TI}$ & $3,254(23.19)$ \\
\hline $\mathrm{T} 2$ & 1,795 (12.79) \\
\hline T3 & $5,132(36.57)$ \\
\hline T4 & $3,852(27.45)$ \\
\hline \multicolumn{2}{|c|}{ Pathologic N-stage } \\
\hline No & $5,682(40.49)$ \\
\hline $\mathrm{N}+$ & $8,35 \mathrm{I}(59.5 \mathrm{I})$ \\
\hline \multicolumn{2}{|l|}{ Age (years) } \\
\hline $10-59$ & $4,538(32.34)$ \\
\hline$\geq 60$ & $9,495(67.66)$ \\
\hline \multicolumn{2}{|l|}{ Sex } \\
\hline Male & $8,676(61.83)$ \\
\hline \multirow[t]{2}{*}{ Female } & $5,357(38.17)$ \\
\hline & $\begin{array}{l}\text { Median (range) of } \\
\text { examined LNs }\end{array}$ \\
\hline \multicolumn{2}{|c|}{ Pathologic T-stage } \\
\hline All patients & $14(1-89)$ \\
\hline TI & $12(1-84)$ \\
\hline T2 & $13(1-89)$ \\
\hline T3 & $15(I-87)$ \\
\hline T4 & $15(1-89)$ \\
\hline
\end{tabular}
disease. Interestingly, this false-negative probability varied

Table I Characteristics of patients included in this study

Abbreviation: LNs, lymph nodes. 
Table 2 Parameters in fitting of beta-binomial model

\begin{tabular}{|l|l|l|l|}
\hline $\begin{array}{l}\text { Pathologic } \\
\text { T-stage }\end{array}$ & $\begin{array}{l}\text { Percent of } \\
\text { simulation }^{\mathrm{a}}\end{array}$ & $\alpha(\mathbf{9 5 \%} \mathrm{CI})$ & $\beta(95 \% \mathrm{CI})$ \\
\hline TI & 20 & $\mathrm{I} .63(\mathrm{I} .34-1.91)$ & $6.08(4.49-7.70)$ \\
\hline T2 & 20 & $1.15(1.02-1.29)$ & $2.95(2.57-3.38)$ \\
\hline T3 & 40 & $1.10(1.04-1.16)$ & $1.70(1.60-1.80)$ \\
\hline T4 & 40 & $1.10(1.04-1.16)$ & $1.04(0.98-1.1 \mathrm{I})$ \\
\hline
\end{tabular}

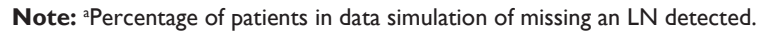
Abbreviation: LN, lymph node.

among patients with different stage. Patients with late $\mathrm{pT}$ stage might suffer from lower risk of false-negative findings as compared with those with early stage (Figure 1A). This result was in accordance with clinical actuality. The apparent prevalence of nodal disease in pT3-4 patients was indeed much higher than in pT1-2 patients (Table 3 ), so the probability of false-negative findings of LN status naturally decreased in patients with pT3-4 stage in comparison with those with pT1-2 stage.

\section{Prevalence of nodal disease adjusted by false-negative findings}

Importantly, the prevalence of nodal disease for GCa patients, adjusted by the false-negative findings, was higher than that of apparent nodal disease. It is independent of the pT stage, indicating that a proportion of patients (adjusted minus apparent prevalence of nodal disease: $5.88 \%, 11.12 \%, 9.37 \%$, and $7.84 \%$ for pT1-4 stages, respectively) were understaged (Table 3). According to the adjusted results, GCa patients would have a high risk of LN metastasis, especially for pT3 and pT4 patients $(81.58 \%$ and $91.02 \%$ for pT3 and pT4, respectively).

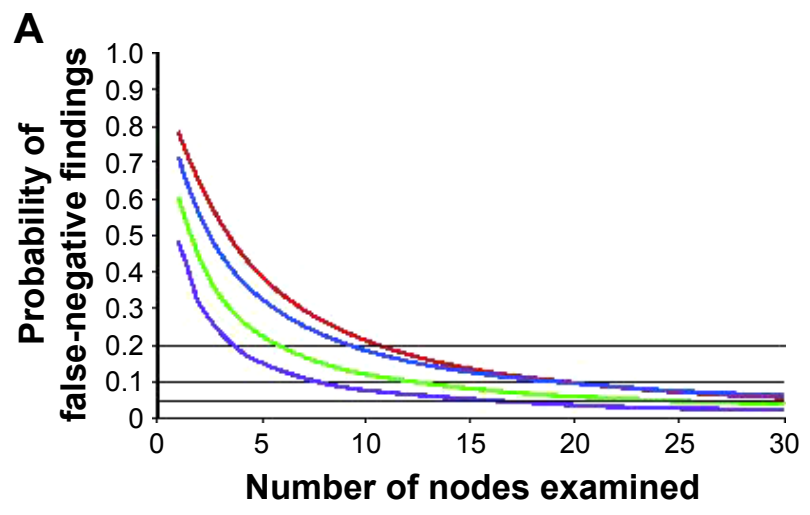

\section{Nodal staging score}

For pT1 patients, NSS was $79.43 \%$, if only a single LN was examined, which increased to $82.61 \%$ and $91.06 \%$, if two and seven LNs were examined, respectively. For pT2 patients, however, 10 and $24 \mathrm{LNs}$ needed to be examined to maintain NSS at the level of $80 \%$ and $90 \%$, respectively. For pT3 and T4 patients, NSS could only be maintained to the levels of $80 \%$, if 23 and $31 \mathrm{LNs}$ were examined, respectively (Figure 1B). In addition, a high NSS was a strong indicator for the prolonged survival in the $\mathrm{pN} 0$ patients across all $\mathrm{pT}$ stages $(P<0.0001$ for all $\mathrm{pT}$ stages, Figure 2$)$, indicating that the adequacy of nodal staging predicted by NSS was associated with patients' survival and can be served as a tool to guide postoperative decision.

\section{Discussion}

To the best of our knowledge, three nodal staging systems for postoperative decision-making have been proposed to date: the pathologic AJCC-based nodal staging system, ${ }^{13}$ the $\log$ odds of positive LNs, ${ }^{14}$ and the LNs ratio,,${ }^{15,16}$ all of which have been demonstrated to be critical indicators for prognosis of GCa patients. Moreover, a series of studies have made great efforts to select a nodal staging system with the best performance in prognostic prediction. ${ }^{17-19}$ Because pN0 patients always have zero-positive LN detected by pathologists, no matter how many LNs are examined, the shared disadvantage of these three nodal staging systems is that they are definitely unresponsive to $\mathrm{pN} 0$ patients, and, therefore, are factually one-size-fit-all nodal staging approaches. Therefore, the major advantage of NSS, compared with the previous nodal staging systems, is that it is responsive to $\mathrm{pN} 0$ patients by quantifying the appropriateness of a

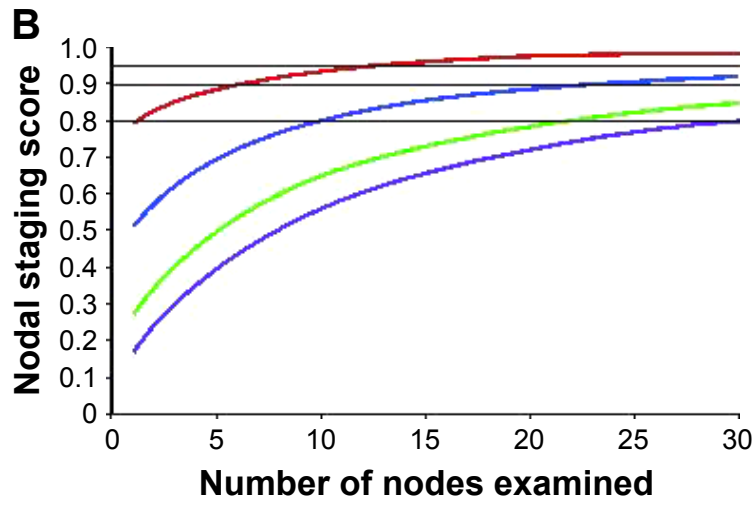

$$
\begin{array}{lll}
\text { T-stage } \\
-1-2-3 & -4
\end{array}
$$

Figure I Using a beta-binomial model to calculate the probability of false-negative findings of LN status, as a function of the number of examined LNs and $\mathrm{pT}$ stage (A). The NSS for pathologic NO GCa patients as a function of the number of examined LNs and PT stage (B).

Abbreviations: GCa, gastric cancer; LN, lymph node; NSS, nodal staging score. 
Table 3 Apparent and adjusted prevalence of nodal disease in GCa with different pathologic T-stage

\begin{tabular}{|l|l|l|}
\hline $\begin{array}{l}\text { Pathologic } \\
\text { T-stage }\end{array}$ & $\begin{array}{l}\text { Apparent } \\
\text { prevalence (\%) }\end{array}$ & $\begin{array}{l}\text { Adjusted } \\
\text { prevalence }^{\text {a }} \text { (\%) }\end{array}$ \\
\hline TI & 18.84 & 24.72 \\
\hline T2 & 46.13 & 57.25 \\
\hline T3 & 72.21 & 81.58 \\
\hline T4 & 83.18 & 91.02 \\
\hline
\end{tabular}

Note: adjusted for the false-negative findings.

Abbreviation: $\mathrm{GCa}$, gastric cancer.

nodal-negative diagnosis. Therefore, for $\mathrm{pN} 0$ patients, NSS is the optimal selection for postoperative LN status evaluation compared with previous staging systems.

Nevertheless, the threshold of LNs that should be removed for GCa patients is still controversial. According to previous studies based on the SEER database, ${ }^{20}$ and a subsequent systematic review, ${ }^{21}$ the total number of LNs resected and examined was a stage-based survival predictor. Although at least 16 LNs examined is the standard recom-
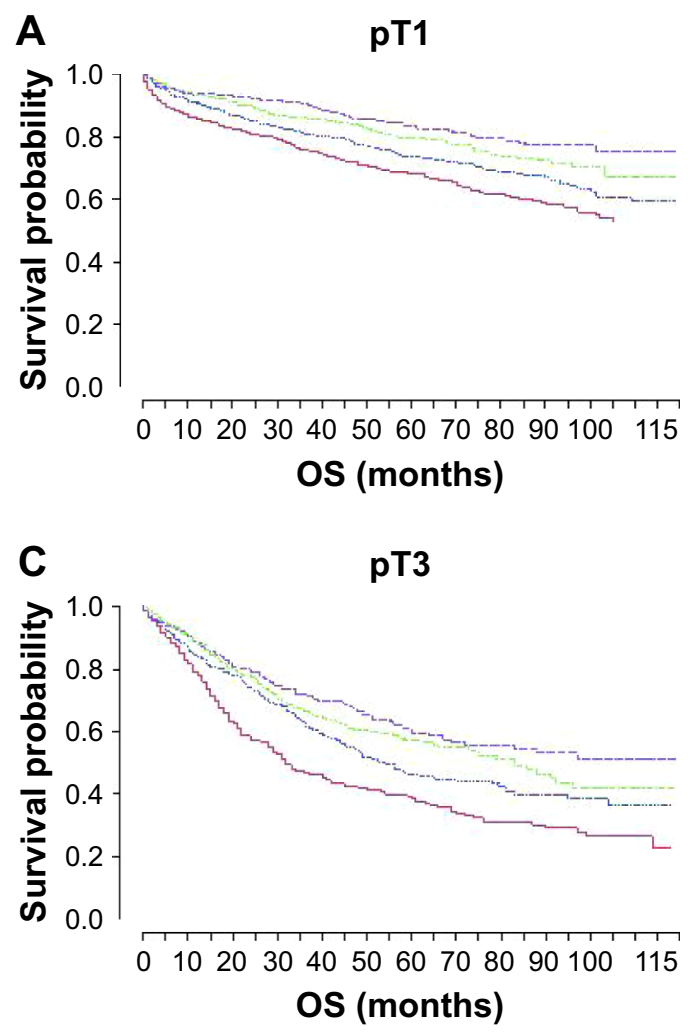

mendation, some studies suggested that more LNs should be examined, ${ }^{22}$ especially for advanced GCa patients. ${ }^{20,21}$ The remarkable finding in the present study is that NSS increased as the number of examined LNs increased and strongly depended on $\mathrm{pT}$ stage. In the modeling, we observed that removing only $7 \mathrm{LNs}$ was enough for an NSS of $90 \%$, which certainly defines that a pT1 patient can be correctly staged as nodal-negative, and for pT2 patients, removing $24 \mathrm{LNs}$ during surgery was enough to reach the same level of NSS. For pT3 and pT4 patients, removing 23 and 31 LNs could also hold the NSS up to $80 \%$, ensuring a high confidence of a correct nodal-negative staging. Of note, patients with pT3-4 stage had a lower NSS as compared with those with pT1-2 stage, indicating a relatively high risk (estimated as 1-NSS) of carrying occult disease in pT3-4 patients if a limited number of LNs were examined. Therefore, our results provide a refined threshold for the number of LNs to be removed for pathologists to determine a true negative diagnosis. Moreover, because patients with late pT stage
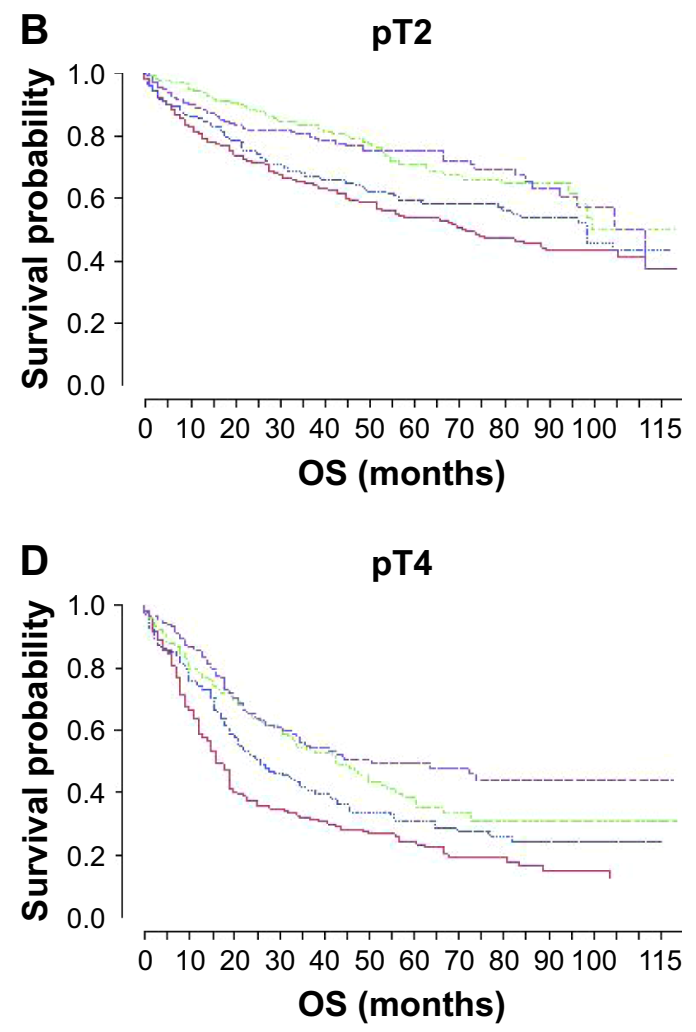

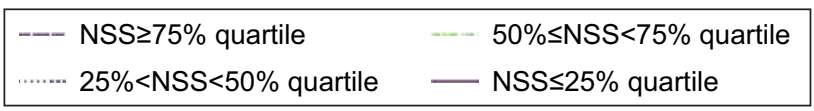

Figure 2 Kaplan-Meier curves assessing the survival rates as a function of the quartile NSS, stratified by the pT stage. The quartile cutoff values were $89.97 \%$, $94.47 \%$, and $96.66 \%$, respectively, for pTI patients (A), $74.79 \%, 82.53 \%$, and $88.53 \%$, respectively, for pT2 patients (B), $57.09 \%, 70.27 \%$, and $78.37 \%$, respectively, for pT3 patients (C), and $43.81 \%, 58.38 \%$, and $69.87 \%$, respectively, for pT4 patients (D).

Abbreviations: NSS, nodal staging score; OS, overall survival. 
have a lower NSS as compared with those with early stage as indicated by our results, we suggest that a substantial proportion of $\mathrm{pT} 3 \mathrm{~N} 0$ and $\mathrm{pT} 4 \mathrm{~N} 0$ patients should receive effective adjuvant therapies to prevent LN recurrence based on NSS. It will be of great interest to observe the results of future clinical trials focusing on novel adjuvant therapeutic strategy for pT3N0 and pT4N0 patients based on NSS.

Because of the lack of effective methods to clarify the true LN status in $\mathrm{pN} 0$ patients, unlike the $\mathrm{pN}+$ patients, the $\mathrm{pT}$ stage was the only way for us to determine the state of disease progression according to the AJCC-based cancer staging system. Importantly, our findings demonstrated that NSS could also help to predict prognosis. Taken together, we have confirmed that the pN0 subpopulation of GCa patients may benefit from NSS in addition to the traditional AJCC-based staging guideline, in terms of either postoperative clinical decision-making or recurrence prevention.

We calculated NSS under the beta-binomial model, ${ }^{23}$ a model that gives the consideration to both binomial distribution and heterogeneity distribution of the $\mathrm{P}(\mathrm{FN})$ among patients. Moreover, to avoid extrapolation to a largest extent, we make a data simulation in the condition of missing an LN, which was randomly assigned and should have been resected during surgery. Therefore, constructing a beta-binomial model using the simulated as a part of dataset could provide a more accurate fitting closer to the real situation where an LN was missed, compared to the previously reported algorism in which data were directly fitted in the model without a simulation. ${ }^{9-12}$ This model was also under an important assumption that there was no false-positive nodal staging in clinical practice. We regard this assumption as reasonable, because pathologists must have substantial expertise and follow pathological guidelines to discriminate a nodalpositive patient. Moreover, a group judgment, instead of single-minded decision, is needed to assert a nodal disease. Therefore, it is statistically and clinically reasonable to compute NSS using false-negative probability derived from beta-binomial model under such an assumption.

Besides, the extranodal extension of nodal metastasis is one of the most important prognostic indicators in GCa. Up to date, a few studies ${ }^{24,25}$ have reported on extracapsular LN involvement in relation to $\mathrm{GCa}$, it demonstrates that extracapsular LN involvement is associated with higher tumor stages and is an independent negative prognostic factor. In future staging systems, not only the number of positive LNs, but also the presence of extracapsular $\mathrm{LN}$ involvement in $\mathrm{GCa}$ should be considered.
The major limitation of the current study is its retrospective design in nature. The missing therapeutic information from the SEER database is another limitation. Multicenter prospective studies with detailed clinical information are needed to validate our findings.

In summary, NSS as a function of number of LNs removed and $\mathrm{pT}$ stage can provide quantified information on adequacy of nodal-negative classification, which is helpful for postoperative decision and patients' consultation on the risk of carrying occult disease. Using this quantified information, we can refine the optimal number of LNs examined for accurate staging. A relatively low level of NSS for pT3N0 and pT4N0 patients points out an unaware problem. Therefore, it is an urgent necessity for clinicians to take an improvement adjuvant therapeutic strategy in comparison with the current standard agents, to prevent the LN recurrence for pT3N0 and pT4N0 patients as illustrated by the NSS-based prediction.

\section{Acknowledgment}

This paper does not contain any studies with human participants or animals performed by any of the authors. This study was funded by National Natural Science Foundation of China (Nos. 81802347).

\section{Disclosure}

The authors report no conflicts of interest in this work.

\section{References}

1. Sakuramoto S, Sasako M, Yamaguchi T, et al. Adjuvant chemotherapy for gastric cancer with S-1, an oral fluoropyrimidine. $N$ Engl J Med. 2007;357(18):1810-1820.

2. Bang YJ, Kim YW, Yang HK, et al; CLASSIC trial investigators. Adjuvant capecitabine and oxaliplatin for gastric cancer after D2 gastrectomy (CLASSIC): a phase 3 open-label, randomised controlled trial. Lancet. 2012;379(9813):315-321.

3. Sasako M, Sakuramoto S, Katai H, et al. Five-year outcomes of a randomized phase III trial comparing adjuvant chemotherapy with S-1 versus surgery alone in stage II or III gastric cancer. J Clin Oncol. 2011;29(33): $4387-4393$.

4. Hundahl SA, Phillips JL, Menck HR. The National Cancer Data Base Report on poor survival of U.S. gastric carcinoma patients treated with gastrectomy: Fifth Edition American Joint Committee on Cancer staging, proximal disease, and the "different disease" hypothesis. Cancer. 2000;88(4):921-932.

5. Smith DD, Schwarz RR, Schwarz RE. Impact of total lymph node count on staging and survival after gastrectomy for gastric cancer: data from a large US-population database. J Clin Oncol. 2005;23(28):7114-7124.

6. de Steur WO, Hartgrink HH, Dikken JL, Putter H, van de Velde CJ. Quality control of lymph node dissection in the Dutch Gastric Cancer Trial. Br J Surg. 2015;102(11):1388-1393.

7. Cao L, Selby LV, Hu X, et al. Risk factors for recurrence in T1-2N0 gastric cancer in the United States and China. J Surg Oncol. 2016; 113(7):745-749. 
8. Zheng WF, Ji TT, Lin Y, Li RZ. The prognostic value of lymph nodes count on survival of patients with node-negative gastric cancer. Oncotarget. 2016;7(28):43680-43688.

9. Robinson TJ, Thomas S, Dinan MA, Roman S, Sosa JA, Hyslop T. How many lymph nodes are enough? Assessing the adequacy of lymph node yield for papillary thyroid cancer. J Clin Oncol. 2016;34(28): 3434-3439.

10. Kluth LA, Abdollah F, Xylinas E, et al. Pathologic nodal staging scores in patients treated with radical prostatectomy: a postoperative decision tool. Eur Urol. 2014;66(3):439-446.

11. Shariat SF, Rink M, Ehdaie B, et al. Pathologic nodal staging score for bladder cancer: a decision tool for adjuvant therapy after radical cystectomy. Eur Urol. 2013;63(2):371-378.

12. Gönen M, Schrag D, Weiser MR. Nodal staging score: a tool to assess adequate staging of node-negative colon cancer. J Clin Oncol. 2009;27(36):6166-6171.

13. Washington $\mathrm{K}$. 7th Edition of the AJCC cancer staging manual: stomach. Ann Surg Oncol. 2010;17(12):3077-3079.

14. Sun Z, Xu Y, Li de M, et al. Log odds of positive lymph nodes: a novel prognostic indicator superior to the number-based and the ratio-based $\mathrm{N}$ category for gastric cancer patients with R0 resection. Cancer. 2010; 116(11):2571-2580.

15. Kutlu OC, Watchell M, Dissanaike S. Metastatic lymph node ratio successfully predicts prognosis in western gastric cancer patients. Surg Oncol. 2015;24(2):84-88.

16. Melis M, Masi A, Pinna A, et al. Does lymph node ratio affect prognosis in gastroesophageal cancer? Am J Surg. 2015;210(3):443-450.
17. Spolverato G, Ejaz A, Kim Y, et al. Prognostic performance of different lymph node staging systems after curative intent resection for gastric adenocarcinoma. Ann Surg. 2015;262(6):991-998.

18. Jian-Hui C, Shi-Rong C, Hui W, et al. Prognostic value of three different lymph node staging systems in the survival of patients with gastric cancer following D2 lymphadenectomy. Tumour Biol. 2016;37(8):11105-11113.

19. Lee JW, Ali B, Park CH, Song KY. Different lymph node staging systems in patients with gastric cancer from Korean: what is the best prognostic assessment tool? Medicine (Baltimore). 2016;95(25):e3860.

20. Schwarz RE, Smith DD. Clinical impact of lymphadenectomy extent in resectable gastric cancer of advanced stage. Ann Surg Oncol. 2007;14(2): $317-328$.

21. Seevaratnam R, Bocicariu A, Cardoso R, et al. How many lymph nodes should be assessed in patients with gastric cancer? A systematic review. Gastric Cancer. 2012;15(Suppl 1):70-88.

22. Huang C. How many lymph nodes should be removed to define an optimal D2 lymphadenectomy for gastric cancer in the modern era? An analysis of 2,947 patients from a two-institution database in China. J Clin Oncol. 2016;34(Suppl 15):4051.

23. Magnussen S, Kremer A. The beta-binomial model for estimating heritabilities of binary traits. Theor Appl Genet. 1995;91(3):544-552.

24. Veronese N, Fassan M, Wood LD, et al. Extranodal extension of nodal metastases is a poor prognostic indicator in gastric cancer: a systematic review and meta-analysis. J Gastrointest Surg. 2016;20(10):1692-1698.

25. Alakus H, Hölscher AH, Grass G, et al. Extracapsular lymph node spread: a new prognostic factor in gastric cancer. Cancer. 2010;116(2): 309-315.
OncoTargets and Therapy

\section{Publish your work in this journal}

OncoTargets and Therapy is an international, peer-reviewed, open access journal focusing on the pathological basis of all cancers, potential targets for therapy and treatment protocols employed to improve the management of cancer patients. The journal also focuses on the impact of management programs and new therapeutic agents and protocols on

\section{Dovepress}

patient perspectives such as quality of life, adherence and satisfaction. The manuscript management system is completely online and includes a very quick and fair peer-review system, which is all easy to use. Visit http://www.dovepress.com/testimonials.php to read real quotes from published authors. 